\title{
Rayleigh like scattering from silica-titania core-shell particles and their application in protection against harmful ultraviolet rays
}

\author{
SUDIPTA G DASTIDAR**, P BHARATH and ARINDAM ROY \\ Unilever Research and Development, Whitefield, Bangalore 560 066, India
}

MS received 20 October 2010; revised 18 January 2011

\begin{abstract}
In this article we report experimental and theoretical results of angle-dependent laser light scattering of nano titanium dioxide nucleated on silica particles. It was observed that the experimental scattering profile from nano-titania coated silica (TCS) particle resembles that of a Rayleigh scattering. It can be inferred from the light scattering profile that nucleating fine particles onto a surface of a bigger particle (core), the resulting scattering profile is dominated by the smaller particles. Thin film transmittance measurement of TCS particles also supports this claim. The theoretical scattering predictions do not match with the experimental findings and the reasons for the discrepancies are addressed. This Rayleigh-like scattering property of TCS particles can be used in cosmetic formulations as a replacement for nanoparticles to provide protection from harmful ultraviolet rays. This study helps to provide insights into these systems for their potential usage in cosmetics.
\end{abstract}

Keywords. Nanoparticles; Rayleigh scattering; transmittance; ultraviolet ray; sunscreen.

\section{Introduction}

In recent years, considerable attention has been devoted to fabricate composite nanostructured materials with functional properties. Core-shell particles are examples of such composite materials where the morphology, microstructure and chemical composition are controlled to achieve various functional properties. Synthesis of various kinds of core-shell particles were reported several decades ago (Iler 1959). The composite particles having silica core and titania shell are widely studied. These particles are typically synthesized using techniques like layer-by-layer deposition (Caruso et al 1999, 2001; Nakamura et al 2005, 2006), sol-gel synthesis (Holgado et al 2000; Guo et al 2003; Kim et al 2003; Liu et al 2005; El-Toni et al 2006; Lim et al 2008), laser pyrolysis (Maskrot et al 2006), heterocoagulation (Wilhelm and Stephen 2006) and flame synthesis (Hu et al 2007). Most of these methods involve multiple steps, $\mathrm{pH}$ control and ingredients like surfactant polyelectrolytes which makes it difficult to replicate in bulk scale. In a recent article, a procedure to synthesize these particles was reported which avoids $\mathrm{pH}$ control and use of ingredients like surfactant, polyelectrolytes, etc (Lim et al 2008). One of the interesting properties of this kind of core-shell particles is their wide range of optical manifestation (Aden and Kerker 1951; Johnson et al 1997; LizMarzan et al 2001; Small et al 2005). The refractive index and the dimensions of the core as well as shell can be va-

*Author for correspondence (ghoshdastidar.sudipta@unilever.com) ried to manipulate the optical properties of these materials. Our interest in this material arises due to its potential usage in personal care products as a sunscreen; thus it is important to understand the light scattering behaviour of these particles.

In this article, we report angle-dependent laser light scattering properties of silica titania core shell particles (TCS) through experimental studies and theoretical predictions. The efficacy of this material to protect against harmful UV rays was evaluated and compared with a commercial nano-titania particle. During the course of the study we also developed a simple process to synthesize TCS particles in bulk scale. Thus, this study would provide an insight into the interaction of light with these kind of particles.

\section{Theory of scattering of light from core-shell particles}

Interaction of electromagnetic radiation with particles is well studied. The classical theory by Mie (1908) gives an analytical solution for scattering from homogenous particles. Theoretical studies to characterize the optical properties of multilayer spherical (Aden and Kerker 1951; Small et al 2005) and planar (Huxley 1968; Land 1972) particles are reported in literature. The theory for light scattering from a coated dielectric sphere, i.e. core-shell particles, was first published by Aden and Kerker (1951). The theory for scattering of electromagnetic waves by core-shell material is discussed in detail elsewhere (Wriedt 2009). The solution under appropriate conditions gives the same expression 
as Mie theory for single sphere. Similar to Mie theory, the scattering cross section $(\sigma)$ is defined as

$$
\sigma=\lim _{r \rightarrow \infty} 4 \pi R^{2} \frac{\left|\vec{E}^{S}\right|^{2}}{\left|\vec{E}_{i}\right|^{2}}
$$

where $R$ is the distance from the scatterer to the receiver, $\vec{E}^{S}$ is the scattered field at the receiver and $\vec{E}^{i}$ is the incident field. The total cross section is the integral of $\sigma$ over the surface of a single sphere. The scattering cross section and the angular distribution of scattered radiation, when a particle is illuminated by a plane wave, can be determined theoretically. The total scattering cross section $(\sigma)$ is expressed as

$$
\sigma=\frac{2 \pi}{\left|K_{3}\right|^{2}} \sum_{n=1}^{\infty}(2 n+1)\left(\left|a_{n}^{2}\right|^{2}+\left|b_{n}^{2}\right|^{2}\right),
$$

where $K_{3}$ is the complex propagation constant in the medium in which the particle is studied. The process of determining the scattering cross section theoretically is extremely laborious. However, algorithms and software are available to perform these calculations. In this article, we used Scatlab 1.2 for theoretical calculations. In this computer code the scattering cross sections $(\sigma)$ and angle-dependent intensities can be calculated for core-shell geometries. The scattering cross section is the ratio of the total scattered power integrated over all directions to the intensity of the incident plane wave on the particle. The scattering cross section is usually converted to scattering efficiency $\left(Q_{\text {scatt }}\right)$ which is the normalized scattering cross section to the geometrical cross section.

$$
Q_{\text {scatt }}=\frac{\sigma}{\pi r^{2}} .
$$

The computational results are discussed in the next section when we discuss the light scattering properties of these materials.

\section{Materials and methods}

\subsection{Materials}

Tetrabutyl orthotitanate (TBOT) and tetraethyl orthosilicate (TEOS) obtained from Aldrich chemicals were used as precursor for the titania shell and silica core, respectively. Analytical grade ethanol (ex. Les Alcohols De Commerce) and ammonia solution (25\%) (ex. Merck) were the other chemicals used during the synthesis process. Milli Q water was used in all the experiments. Nano-titania particles from Degussa $\left(\mathrm{P} 25, \mathrm{TiO}_{2}\right)$ was used as a model commercial particle to compare the efficacy of protection in UV region.
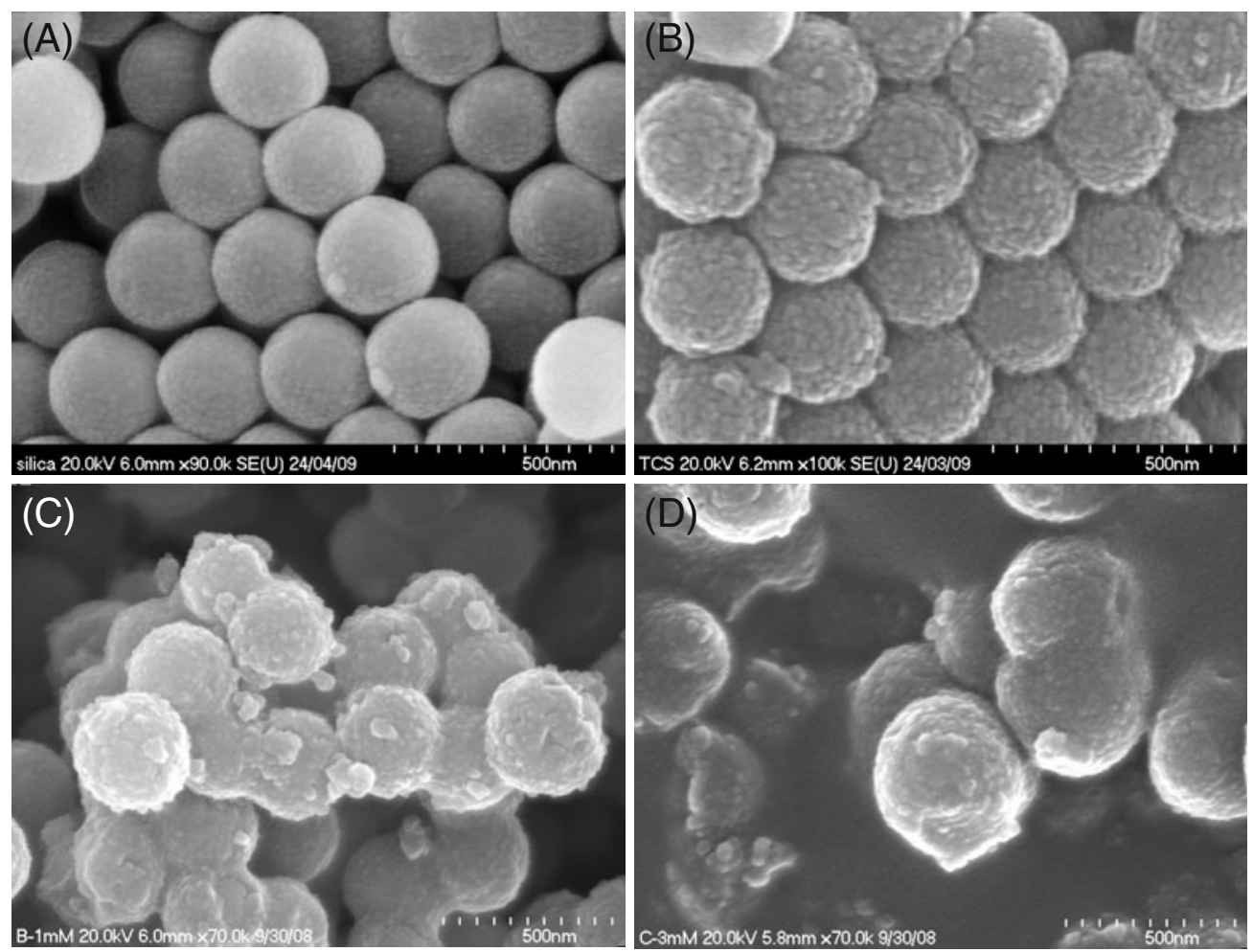

Figure 1. Scanning electron micrographs of (A) silica and TCS particles synthesized using (B) $0.6 \mathrm{mmol}$, (C) $1 \mathrm{mmol}$, and (D) $3 \mathrm{mmol}$ of TBOT. 


\subsection{Synthesis of silica core}

Silica spheres were synthesized using classical Stober's process (Stober et al 1968). Eight millilitres of 25\% ammonia, $147 \mathrm{ml}$ of ethanol and $5 \mathrm{ml}$ of Milli Q water were taken in a $500 \mathrm{ml}$ conical flask and the solution was stirred for $30 \mathrm{~min}$ using a magnetic stirrer. To this solution $7.2 \mathrm{ml}$ of TEOS was added dropwise under continuous stirring. The reaction was continued for $8 \mathrm{~h}$ and the particles were collected by centrifugation (ex. Remi R4-C Laboratory centrifuge) at $3000 \mathrm{rpm}$ for $30 \mathrm{~min}$. The particles obtained were washed twice with ethanol and dried at $40^{\circ} \mathrm{C}$.

\subsection{Synthesis of titania-coated silica particles}

Synthesized silica particles $(250 \mathrm{mg}$ ) were dispersed in $50 \mathrm{ml}$ of ethanol through sonication (ex. Microsupersonics) in a round bottom (RB) flask. To this suspension, $0.5 \mathrm{ml}$ of Milli $\mathrm{Q}$ water was added and the suspension was stirred using magnetic stirrer. The RB flask was fitted with a condenser and water was circulated throughout the synthesis. This suspension was heated to $70^{\circ} \mathrm{C}$ followed by dropwise addition of TBOT. The reaction was performed with different concentrations $(0 \cdot 6,1$ and $3 \mathrm{mmol})$ of TBOT to obtain different shell thicknesses. The reaction was continued for $2 \mathrm{~h}$ and the suspension was centrifuged (at $3000 \mathrm{rpm}$ for $30 \mathrm{~min}$ in a Remi R4-C Laboratory centrifuge) to collect the particles, washed twice with ethanol and dried at $40^{\circ} \mathrm{C}$. In another set of experiment, the reaction time was varied from $0.5,1,1.5$ and $2 \mathrm{~h}$ for the fixed concentration $(0.6 \mathrm{mmol})$ of TBOT. The amorphous titania-coated silica particle thus obtained was calcined in a muffle furnace (ex. Thermolyn 48000 ) at $700^{\circ} \mathrm{C}$ for $5 \mathrm{~h}$.

\subsection{Particle characterization}

Scanning electron micrographs of synthesized silica and titania-coated silica particles were collected using a HITACHI S-4700 scanning electron microscope (SEM) and transmission electron micrographs were collected using a Philips CM 200 microscope (TEM). The SEM and TEM images were subjected to image analysis using Image proplus software (Version 4.1) to determine the particle size. Energy dispersive X-ray analysis (EDAX) was performed on these particles using HORIBA EMAX X-ray analyzer to determine the chemical composition of the materials. The crystallinity of the samples was determined using a Siemens D5000 X-ray diffractometer. Particle size of both silica and TCS were determined in ethanol medium through dynamic laser light scattering set up from Brookhaven Corporation using Lexel 95 laser $(\lambda=488 \mathrm{~nm})$.

\subsection{Static light scattering (SLS) measurements}

The angle-dependent scattering intensity, $I(\theta)$, of both silica and TCS particles were measured using BI-200SM
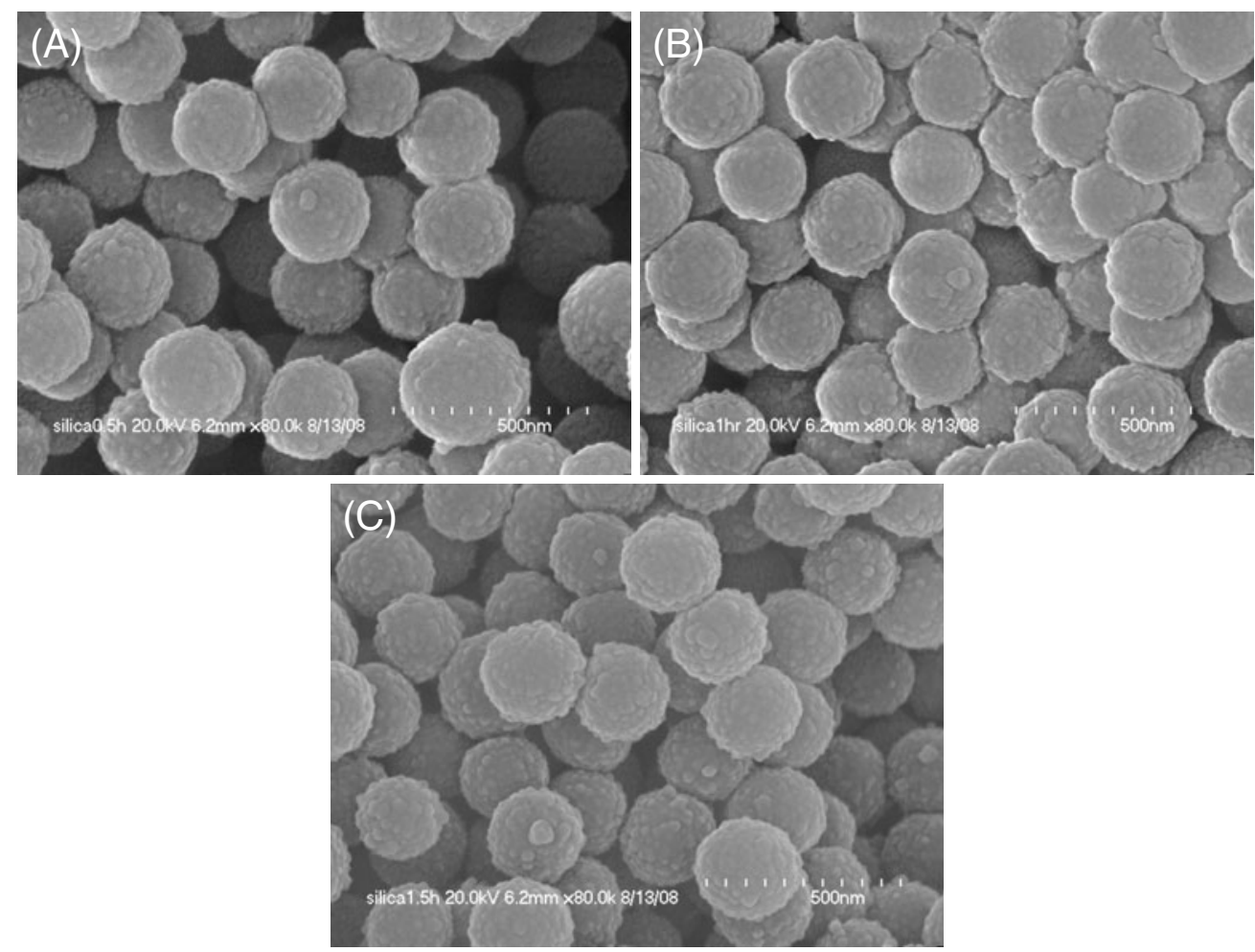

Figure 2. Scanning electron micrographs of TCS particles synthesized using $0.6 \mathrm{mmol}$ TBOT at various time intervals: (A) $0 \cdot 5$, (B) 1 , and (C) $1.5 \mathrm{~h}$. 
goniometer set up from Brookhaven Corporation using Lexel 95 Laser $(\lambda=488 \mathrm{~nm})$. In order to avoid the multiple scattering, a dilute suspension of the particles $(0.001,0.005$, $0.01 \mathrm{~g} / \mathrm{l}$ ) were prepared in ethanol (by sonication) and used for analysis. Scattering profiles were collected from $25^{\circ}$ to $155^{\circ}$ and SLS experiments were repeated several times to obtain consistency. The theoretical light scattering profile resulting from a single TCS and silica particle were computed using an algorithm available as a freeware (Scatlab Verion 1.2) and was compared with experimental result.

\subsection{Thin film transmittance measurements}

An industry-recognized protocol was followed to determine the efficacy of the material to provide protection against UV rays. Transpore tape (ex. $3 \mathrm{M}$ ) was used as a substrate to assess the efficacy of TCS particles and commercially available titania nanoparticles (ex. Degussa P-25, average primary particle size is $21 \mathrm{~nm}$ as mentioned by the supplier). Both TCS and Degussa P25 particles were dispersed separately in dipropylene glycol (ex. Aldrich), at $1 \%$ titania level (w/v) via sonication (30 min) and was used for the measurements. The transpore tape was stretched on a sample holder and concentration of $2 \mathrm{mg} / \mathrm{cm}^{2}$ of the sample was dispensed uniformly using a syringe and the sample was spread uniformly using a parafilm (ex. Pechiney Plastic Packaging, USA). The film was allowed to dry for $15 \mathrm{~min}$ and the transmittance spectrum was collected using SPF-290S (ex. Optometrics Corporation, USA) spectrophotometer. The instrument collects transmittance spectrum at six different spots for a given sample. The experiment was repeated for three times and the data reported is thus an average of 18 data points. The reference transmittance scan was obtained using a blank plate, with no sample on transpore tape.

\section{Results and discussion}

\subsection{Synthesis optimization}

Concentration of the precursor (TBOT) and time of the reaction were the parameters optimized for the synthesis for a fixed concentration of silica particles. SEM images of the silica and TCS particles prepared using 0.6, 1 and $3 \mathrm{mmol}$ of TBOT are presented in figure 1 . It can be inferred from the scanning electron micrographs that higher concentration of TBOT ( 1 and $3 \mathrm{mmol}$ ) results in non-uniform nucleation of titania and also the particles appear to be sintered. The TCS particles synthesized using $0.6 \mathrm{mmol}$ of TBOT on the other hand resulted in a uniform nucleation and were also less sintered. The other parameter, reaction time was varied from $0.5,1,1.5$ and $2 \mathrm{~h}$ for the optimized concentration of $0.6 \mathrm{mmol}$ of TBOT. The SEM images of time-dependent reactions are presented in figure 2 and it was observed that the variation of time does not result in significant differences in the morphology of TCS particles. However, to ensure completion of the titania nucleation process the reaction time was maintained for $2 \mathrm{~h}$. Thus, the TCS particles prepared by the addition of $0.6 \mathrm{mmol}$ of TBOT and reaction time of $2 \mathrm{~h}$ were fixed for further studies. Thus, this process does not involve any complicated steps and is highly reproducible which can be utilized to produce TCS particles in a bulk scale.

\subsection{Particle characterization}

The SEM images of both silica and TCS particles are presented in figure 1. The SEM (figure 1) and TEM (figure 3) images coupled with image analysis indicate that the silica particles are $\sim 260 \mathrm{~nm}$ in diameter with titania particles of size $\sim 20 \mathrm{~nm}$ nucleated on the silica surface. The EDAX performed on the TCS particles also indicates the presence of titania on the silica surface. The X-ray diffractogram (XRD) obtained for TCS particles has a primary peak at a $2 \theta$ value of $25.4^{\circ}$ which indicates the presence of anatase phase of
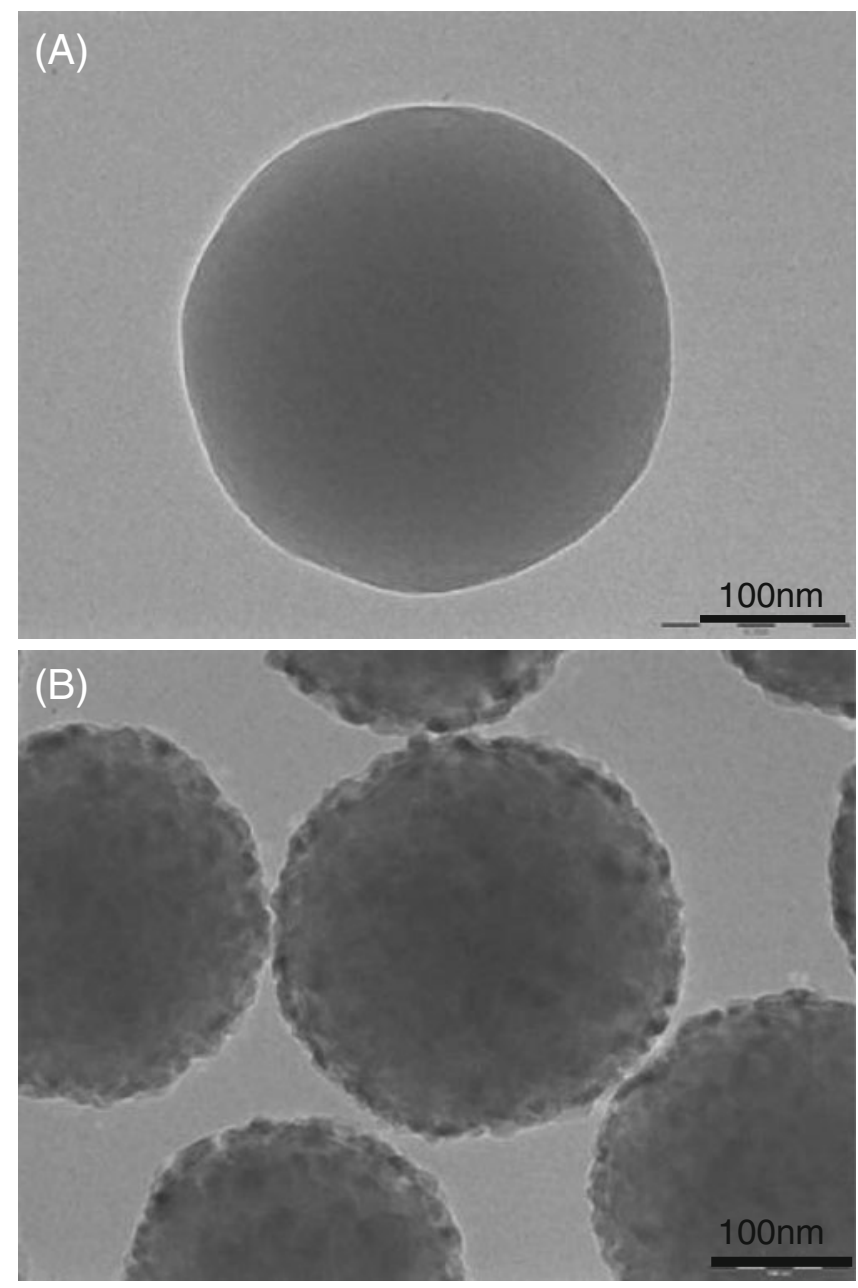

Figure 3. Transmission electron micrographs of (A) silica, and (B) TCS particles prepared using 0.6 mmol of TBOT for $2 \mathrm{~h}$. 
titania. Particle size distribution measured using dynamic light scattering (DLS) technique for both silica and core-shell particles indicates that size of the silica particles is $\sim 260 \mathrm{~nm}$ and that of TCS particles is $\sim 298 \mathrm{~nm}$. The calculated shell thickness from DLS measurement is $\sim 20 \mathrm{~nm}$, which is in agreement with the TEM measurements. The DLS measurement also indicates that TCS particles are highly monodisperse.
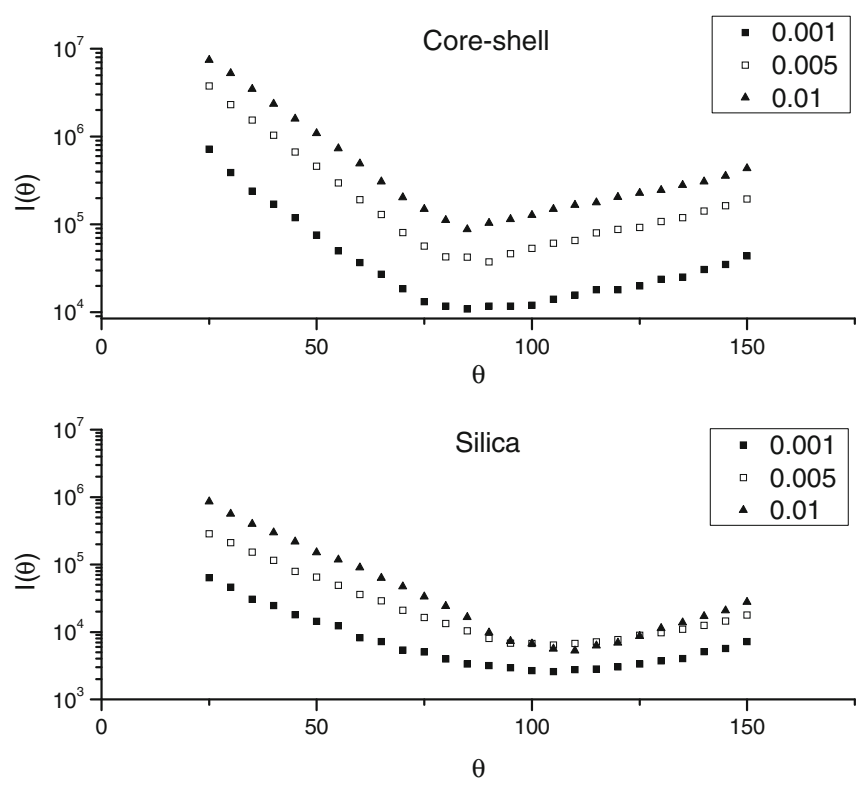

Figure 4. Static laser light scattering intensity as a function of the detector angle and concentrations for silica and TCS particles. The concentrations indicated in the legend are in $\mathrm{g} / \mathrm{l}$.

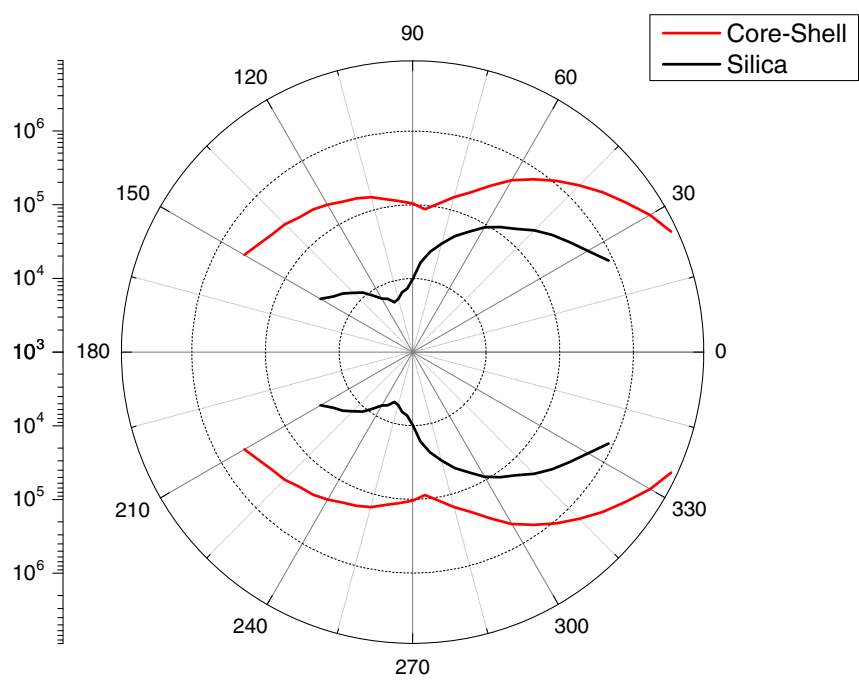

Figure 5. Experimental static laser light scattering $(\lambda=488 \mathrm{~nm}$ laser) intensity as a function of detector angle plotted as a polar diagram for silica and TCS particle for a concentration of $0.01 \mathrm{~g} / \mathrm{l}$.

\subsection{Static light scattering}

The experimental scattered intensity, $I(\theta)$, as a function of detector angle $\theta$ of silica and TCS particles at various concentrations are presented in figure 4 . The concentrations used for light scattering experiment are low enough to avoid multiple scattering. An increase in the concentration of particles resulted in increased scattered intensity which is due to

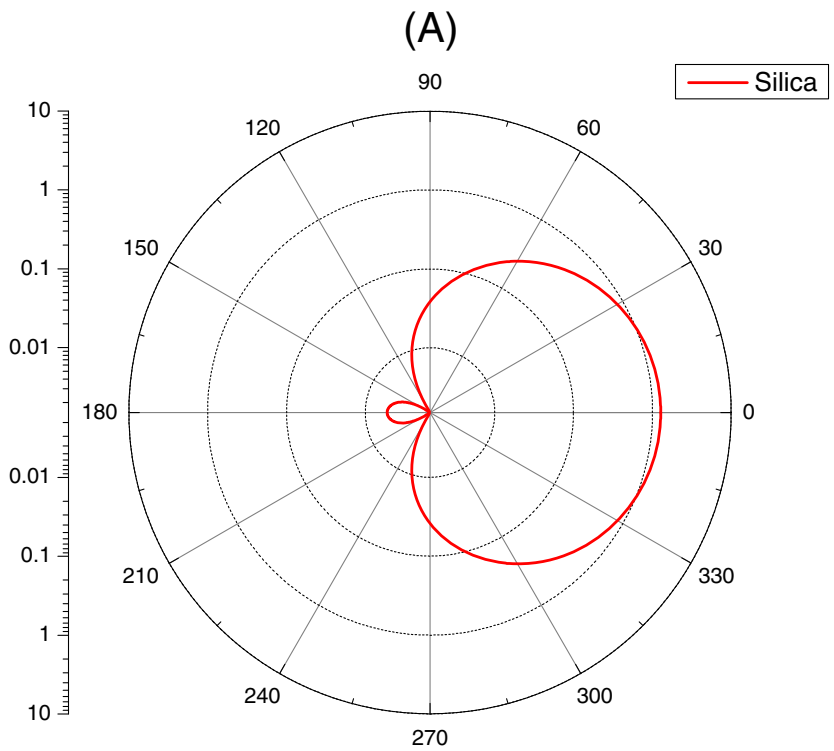

(B)

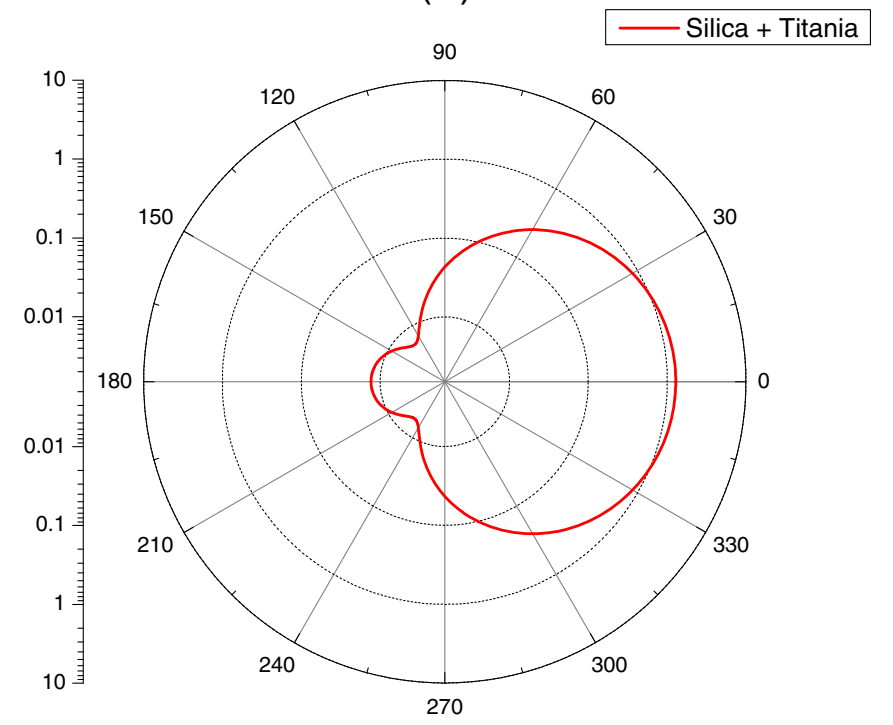

Figure 6. Theoretical scattered intensity as function of angle for the unpolarized incident light (for $\lambda=488 \mathrm{~nm}$ ) plotted as a polar diagram for (A) silica $(260 \mathrm{~nm})$ and (B) silica core (260 nm)-titania shell $(20 \mathrm{~nm})$ particle in ethanol medium. The refractive indices used for the simulation was $1.5,2.5$ and 1.37 for silica, titania and ethanol, respectively. 
the presence of more scatterers at higher concentration levels. This increase of scattering as a function of concentration indicates that multiple scattering is avoided. The scattered intensity of one of the representative concentrations of silica and TCS particles $(0 \cdot 01 \mathrm{~g} / \mathrm{l})$ are presented as polar plots in figure 5. The scattered intensity obtained for TCS particle is higher than that of silica particle at same concentration due to the presence of titania (high refractive index) on silica surface. The theoretical scattering profile for silica of size $260 \mathrm{~nm}$ interacting with an incident radiation of wavelength, $488 \mathrm{~nm}$, was computed using Scatlab (version 1.2) and is presented in figure 6(a). The overall theoretical profile computed matches very well with the experimental result for silica particles. Theoretically a silica particle of size $260 \mathrm{~nm}$ diameter interacting with an incident radiation of $488 \mathrm{~nm}$ gives a size parameter $(\chi)$ of 1.67 (where $\chi=2 \pi a / \lambda$, $a$ is the radius of the particle and, $\lambda$ the wavelength of the incident light in the surrounding medium) (Hulst van de 1981; Bohren and Huffmann 1998) and this results in a scattering ratio of $\sim$ : 1 (forward scattering : backward scattering). The experimental results also exhibited similar ratios. In addition to this, for particles whose size is very small compared to wavelength of light the scattering minima occurs at $90^{\circ}$ but in the case of silica particles it occurs at $\sim 100^{\circ}$ which is probably due to an increased size parameter that shifts the peak of polarization angle towards the higher side.

The experimental scattering profile of TCS particle $(0.01 \mathrm{~g} / \mathrm{l})$ is presented in figure 5. In case of TCS particles, we expected that there would be an increased forward and lesser backward scattering (higher forward to backward scattering

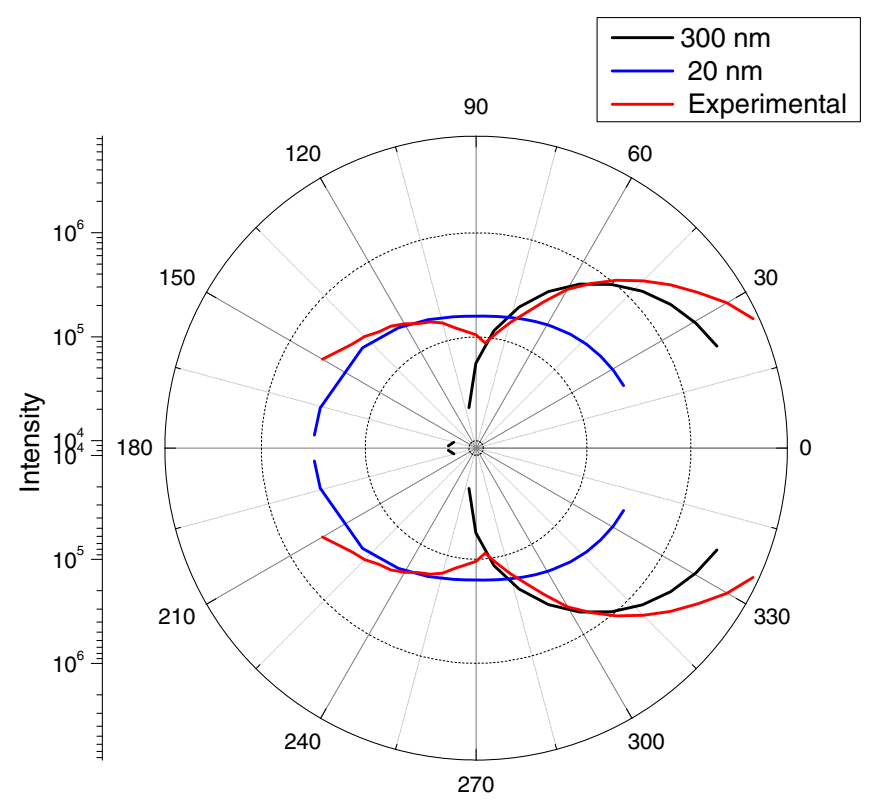

Figure 7. Theoretical light scattering polar plot using Rayleigh equation for a titania particle of size $20 \mathrm{~nm}$ and $300 \mathrm{~nm}$ and experimental scattering profile obtained for TCS particle with a concentration of $0.01 \mathrm{~g} / \mathrm{l}$. ratio) compared to silica particles due to the increased size parameter as a result of the coating. However, the experimental result was completely contrary to what we expected. The scattering profile has equal amount of backward and forward scattering with a minimum at $\sim 90^{\circ}$. This kind of scattering profile can only be obtained for particles in the Rayleigh domain (size extremely small compared to wavelength of light). The theoretical light scattering profile for this particle computed using Scatlab predicted the same higher forward scattering but lesser back scattering (figure 6(b)) as expected from classical scattering theories (Hulst van de 1981). In this respect, the experimental and theoretical results did not match. It is also well known that the equal forward and backward scattering ratio can only be obtained when particle size is very small compared to the wavelength of light, but not from bigger size particle like TCS, which has an average size of $\sim 300 \mathrm{~nm}$. According to our study, the only possible way to obtain Rayleigh kind of scattering profile from TCS particle is when the individual titania particles $(\sim 15-20 \mathrm{~nm})$ nucleated on to the surface of silica particle acts as a scattering centre for the incoming incident radiation. The fact that titania particles nucleate as individual nanoparticles is evident from the SEM (figure 1) and TEM (figure 3) micrographs. In this context we also compared the experimental light-scattering data with pure Rayleigh scattering and the results are presented in figure 7 . The experimental light scattering data was fitted with $300 \mathrm{~nm}$ and $20 \mathrm{~nm}$ titania particles. A $300 \mathrm{~nm}$ titania particle was used as the combined geometrical size of the silica with nucleated titania particles amounts to $300 \mathrm{~nm}$. The $20 \mathrm{~nm}$ titania particle was used in numerical fit because the experimentally determined size of the titania nucleate is $\sim 20 \mathrm{~nm}$. It was observed that

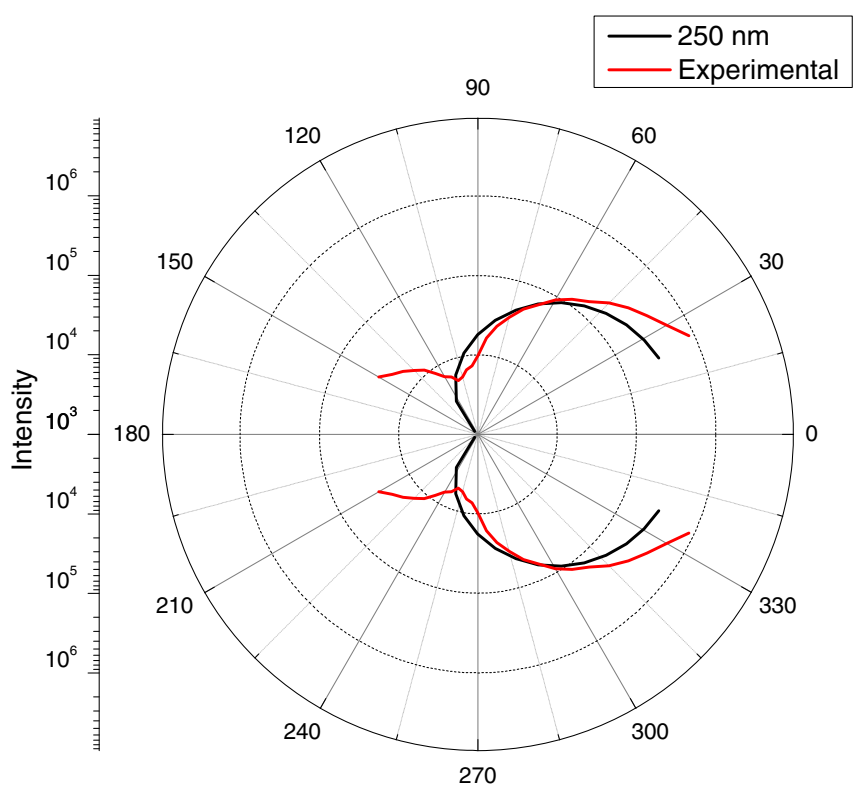

Figure 8. Theoretical light scattering polar plot using Rayleigh equation for a silica particle of size $250 \mathrm{~nm}$ and experimental scattering profile obtained with a concentration of $0.01 \mathrm{~g} / \mathrm{l}$. 
only back scattering profile of the $20 \mathrm{~nm}$ particles matches with the back scattering trend of the experiment but discrepancy was observed in their forward scattering region. The simulation results of $300 \mathrm{~nm}$ titania also did not match as expected because a bigger size particle would not fit in Rayleigh domain. Thus the simulation result of $20 \mathrm{~nm}$ as well as $300 \mathrm{~nm}$ did not match with the experimental data. So, it can be inferred from Mie simulation (Scatlab) as well as Rayleigh model that scattering from the TCS particle is neither exclusively due to the surface titania nucleates nor due to its bigger geometry, but it could be a combination of both dominated by surface scattering.

As mentioned above the experimental light scattering pattern of silica matches well with the theoretical prediction using Scatlab (figure 6(a)). However, as expected when we used pure Rayleigh equation and fit the experimental data for silica (figure 8), there was a mismatch. It was observed that the forward scattering profile matches to some extent whereas the backward scattering did not match with the experimental data. This discrepancy was also expected as Rayleigh scattering is used to explain scattering from extremely small particles $(r \ll \lambda)$. This condition is not met for silica particle because of its size parameter.

It is known from literatures that UV rays induce significant damage to the skin, for example, sunburn, inflammation, cancer, etc hence it is essential to protect skin from UV radiation. In most of the cosmetic and sunscreen products, particles of very fine size are used to protect against harmful UV rays (Lowe et al 1997). This is because of their excellent UV attenuation behaviour compared to their larger homologues. In recent years, use of nano particles in cosmetics and related products is under serious consideration because of their toxicity associated with inhalation, penetrations, etc. (Nohynek et al 2008; Suh et al 2009). If a relatively bigger particle can exhibit scattering pattern similar to that of nanoparticle, it can overcome the safety-related issues because of its size. TCS particle exhibits a scattering pattern similar to

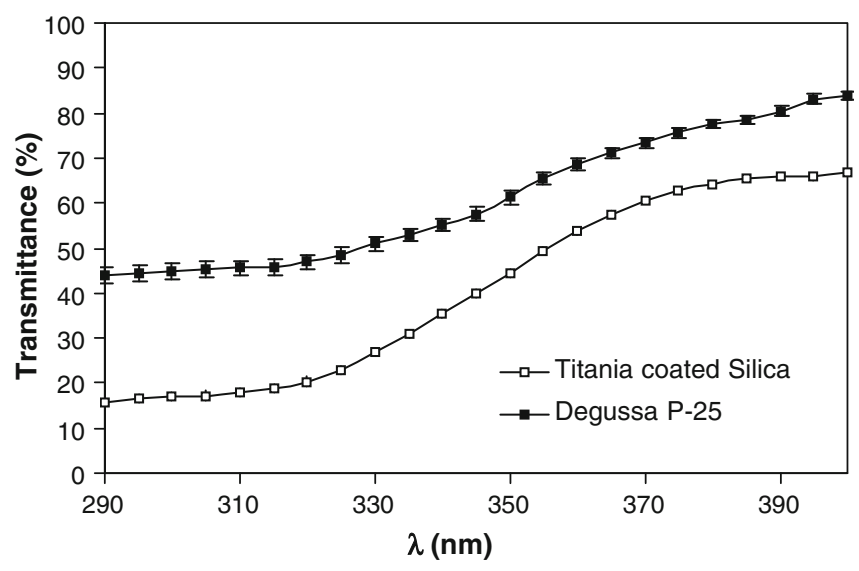

Figure 9. Transmittance spectrum of TCS particles ( $\square$ ) and commercial titania particles ( $\mathbf{\square})$. Both experiments were performed at matched titania levels.
Rayleigh and thus can serve as physical blocker in sunscreen products.

\subsection{Thin film transmittance}

Thin film transmittance spectra of commercial nano-titania particle and TCS particles are presented in figure 9. The measurements were performed at matched titania concentrations of $1 \%(\mathrm{w} / \mathrm{v})$. It was observed that the spectral pattern obtained from Degussa nanoparticle and TCS particles were similar in the UV range. The results also indicate that the TCS particles provide enhanced protection from UV rays (lesser transmittance) compared to commercial nanoparticles at matched titania concentrations. Though the quantity of titania present on surface of the silica particle is less, it still demonstrates the proof of principle to use them as physical blockers.

\section{Conclusions}

Angle-dependent laser light scattering properties of nanotitania nucleated silica particle were studied and were compared with theoretical predictions. It was observed that scattering profile from titania-coated silica particle resembles that of scattering in the Rayleigh domain. The theoretical prediction did not match with the experimental findings and the discrepancy between theory and experiment could be due to the scattering from the surface titania nucleates. It can be inferred from light scattering profile that if fine particles are nucleated on surface of a bigger particle (core), the scattering profile is dominated by the smaller surface particles which leads to Rayleigh like scattering. Thin film transmittance experiment shows that these particles can provide protection from harmful UV rays similar to nanoparticles. This kind of scattering profile from a relatively bigger-sized particle opens up avenues to use them in cosmetic formulations as sunscreens.

\section{Acknowledgements}

The authors would like to acknowledge $\mathrm{Mr}$ Madrid Johnson and Dr Venkat Shankar Iyer of Measurement Science Division at Unilever Research and Development India, Bangalore for performing SEM and XRD measurements, respectively. The TEM measurements were performed at Sophisticated Analytical Instrumental Facility at Indian Institute of Technology Bombay, Mumbai, India. Our sincere gratitude to Dr Janhavi Raut for help in simulation of these systems and Dr Ram Rajagopal for helpful discussions.

\section{References}

Aden A L and Kerker M 1951 J. Appl. Phys. 221242 
Bohren C F and Huffmann D R 1998 Absorption and scattering of light by small particles (NY: Wiley Interscience Publishers)

Caruso F, Susha A, Giersig M and Mohwald H 1999 Adv. Mater. 11 950

Caruso R A, Susha A and Caruso F 2001 Chem. Mater. 13400

El-Toni A M, Yin S and Sato T 2006 J. Colloid Interf. Sci. 300123

Guo C W, Cao Y, Xie S H, Dai W L and Fan K N 2003 Chem. Commun. 6700

Holgado M, Cintas A, Ibisate M, Serna C J, López C and Meseguer F 2000 J. Colloid Interf. Sci. 2296

Hulst van de H C 1981 Light scattering by small particles (NY: Dover Publications)

Huxley A F 1968 J. Exp. Biol. 48227

Hu Y J, Li C Z, Gu F and Zhao Y 2007 J. Alloy Compd. 432 L5

Iler R K 1959 U.S. Patent 2,885, 366

Johnson R W, Thiele E S and French R H 1997 Tappi J. 80233

Kim K D, Bae H J and Kim H T 2003 Colloid Surf. A221 163

Land M F 1972 Prog. Biophys. Mol. Biol. 2477

Lim S H, Phonthammachai N, Pramana S S and White T J 2008 Langmuir 246226

Liu L, Dong P, Liu R, Zhou Q, Wang X, Yi G and Cheng B 2005 J. Colloid Interf. Sci. 2881
Liz-Marzan L M, Correa-Duarte M A, Pastoriza-Santos I, Mulvaney I, Ung T and Geiersig M 2001 in Handbook of surfaces and interfaces of materials (eds) N A Kotov and H S Nalwa (NY: Academic Press) Vol. 3, p. 189

Lowe N J, Shaath N A and Pathak M A (eds) 1997 Sunscreen (NY: Marcel Dekker)

Maskrot H, Herlin-Boime N, Leconte Y, Jursikova K, Reynaud C and Vicens J 2006 J. Nanopart. Res. 8351

Mie G 1908 Ann. Phys. (Leipzig) 330377

Nakamura H, Ishii M, Tsukigase A, Harada M and Nakano H 2005 Langmuir 218918

Nakamura H, Ishii M, Tsukigase A, Harada M and Nakano H 2006 Langmuir 221268

Nohynek G J, Dufour E K and Roberts M S 2008 Skin Pharmacol. Physiol. 21136

Small A, Hong S and Pine D 2005 J. Polym. Sci. Polym. Phys. 43 3534

Stober W, Fink A and Bohn E 1968 J. Colloid Interf. Sci. 2662

Suh W H, Suslick K S, Stucky G D and Suh Y H 2009 Prog. Neurobiol. 87133

Wilhelm P and Stephen D 2006 J. Colloid Interf. Sci. 29388

Wriedt T 2009 J. Quant. Spectrosc. RA 110833 